\title{
Reducing the stigma of public health messages. Invited commentary on ... . Everybody gets stressed ${ }^{\dagger}$
}

\section{SUMMARY}

Human beings have evolved the capacity to classify everything they perceive and our urge to categorise psychological phenomena is little

\author{
different. Categories make life \\ easier to manage. However, \\ nature rarely comes wrapped \\ in discrete packages, and as \\ David Kingdon persuasively
}

\author{
argues in his editorial, we might do \\ well to avoid the catch-all term \\ 'mental disorder' in our public health \\ messages.
}

\section{What is a mental disorder?}

There is often no valid and repeatable dividing line between so-called normality and mental disorder. But it is not only in our public messages that psychiatric diagnosis can be challenged. Mental disorder is a stigmatising term ${ }^{1}$ that rarely captures the complexity of our striving to find meaning in the face of pain and loss. ${ }^{2}$ Moreover, definitions of mental illness which are based on impairment of daily functioning are not particularly accurate: someone performs normally in their work, family life and hobbies until the day they place a rope around their neck; another takes off long periods from work for what we might regard as mild anxiety. Nor does a list of symptoms necessarily get us out of this fix: hallucinations are my rich religious experience while they are your signs of a serious illness. Although the same criticisms may be levelled at the concept of physical disorder, at least here we have physical signs and laboratory measures that allow some sort of validity. A diastolic blood pressure over 90 has validity because research shows that it is closely related to future physical morbidity. But in many psychological disturbances we have little to go on beyond the patient's behaviour and reported mental state, both of which may have little predictive validity.

Finally, psychiatric diagnoses have a poor record when it comes to longevity; over the years they come and go from international classifications, sometimes more at the whim of cultural values, fashion and the profitdriven motives of the pharmaceutical industry than on the basis of empirical evidence. Recent revelations about the links between leading psychiatrists and pharmaceutical companies are not reassuring for the profession or the public. ${ }^{3,4}$

The most common field where psychiatric diagnostic categories run into difficulty is general practice. Family doctors rarely make a specific diagnosis of major depression before prescribing antidepressants. Rather, they go by a gut feeling based on the patient's complaints and their preparedness to accept treatment. ${ }^{5}$ Furthermore, diagnosing and treating are sometimes ways in which general practitioners avoid addressing the complex difficulties that patients experience in their lives and the lives of those close to them. This is not an example of bad practice; rather it is one of real-world practice where categories are unhelpful.

\section{Stress model in place of mental disorder}

So at one level we should welcome David Kingdon's exhortation to replace mental disorder with a stress model that can include us all. Human existence is a struggle to compete and prosper, and stress is a useful blanket description of the pressures we are under. How we react to those pressures, both physically and psychologically, depends on our background, culture and constitution. This familiar description of life has been the focus of our long history of artistic endeavour. A multitude of stories, novels, plays and films has at their heart the human reaction to stress. Moreover, contextualising mental pain makes it understandable. ${ }^{6}$

\section{Humans and machines}

But there also is a potential downside to his idea. The stress model Kingdon recommends is somewhat reductionist and mechanical. Human beings are physical objects subjected to stress and strain; they come under attack and as inflexible physical structures there may be no way out. To avoid such mechanics, the model needs to regard the person as an active, engaged, determining being, rather than a malfunctioning machine. ${ }^{5}$

It does not end there; resorting to categories rather than dimensions is also common in research. In reporting on cohorts or randomised trials we often prefer outcomes couched in terms of numbers of people 'recovered' rather than degree of recovery. Epidemiologists like me count 'heads' and often express their findings in terms of numbers of people falling ill. For example, we recently developed a risk prediction algorithm to identify people at risk of future episodes of major depression over one year. ${ }^{7}$ Although we found that it was as accurate as risk algorithms for onset of cardiac disease, we could just as easily have predicted degree of depressive phenomena by dividing people up into cases or non-cases. Furthermore, when we examined risk of future anxiety (as a category), the algorithm developed came extremely close to that originally developed for major depression. This suggests extensive overlap between these two so-called illnesses. It does not mean we cannot accurately predict mental states. It just means we do not have to cut them at thresholds. 
an

editorial

\section{Creating a self-obsessed nation?}

I do not share, however, David Kingdon's concerns that a stress model is likely to make the nation either more narcissistic or liable to recommend 'pulling yourself together'. These tendencies already exist and categorisation makes them worse. Homosexuals make heterosexuals feel superior. I may be overweight but at least I am not obese. Middle-aged, yes, but at least I am not a pensioner. As Kingdon says, if we are all subject to stress, then our so-called mental illnesses are just degrees of reaction. This dimensional model does not mean, however, that all mental events are purely reactions to stress. Like all things in nature, environment interacts with biology. In some cases, constitution may predominate; in others, the environment. So I would endorse Kingdon's call to avoid the word 'disorder' in our public discourse and our efforts at prevention. It is inaccurate, often adds little to the decision to treat and because of stigma, offers an uncertain shelter for those who want to avoid life for a while.

\section{References}

Thornicroft G, Rose D, Kassam A Sartorius N. Stigma: ignorance, prejudice or discrimination? BrJ Psychiatry 2007; 190: 192-3.

2 FrankIV. Man's Search for Meaning. Rider, 2004.

3 Cosgrove L, Bursztajn HJ, KrimskyS, Anaya M, Walker J. Conflicts of interest and disclosure in the American Psychiatric Association's Clinical Practice Guidelines. Psychother Psychosom 2009; 78 : 228-32.

4 Cosgrove L, KrimskyS, Vijayaraghavan M, Schneider L. Financial ties between DSM-IV panel members and the pharmaceutical industry. Psychother Psychosom 2006: 75: 154-60.
5 Dowrick C. Beyond Depression: A NewApproach to Understanding and Management. Oxford University Press, 2004

6 Horwitz AV, Wakefield JC. The Loss of Sadness. How PsychiatryTransformed Normal Sorrow into Depressive Disorder. Oxford University Press, 2007

7 King M, Walker C, Levy G, Bottomley C, Royston P, Weich S, et al. Development and validation of an international risk prediction algorithm for episodes of major depression in general practice attendees: the PredictD study. Arch Gen Psychiatry 2008; 65 1368-76.
Michael King Professor of Primary Care Psychiatry and Co-Director of PRIMENT Clinical Trials Unit, Department of Mental Health Sciences, University College London Medical School, Royal Free Campus, Rowland Hill Street, London NW32PF, email:m.king@medsch.ucl.ac.uk 